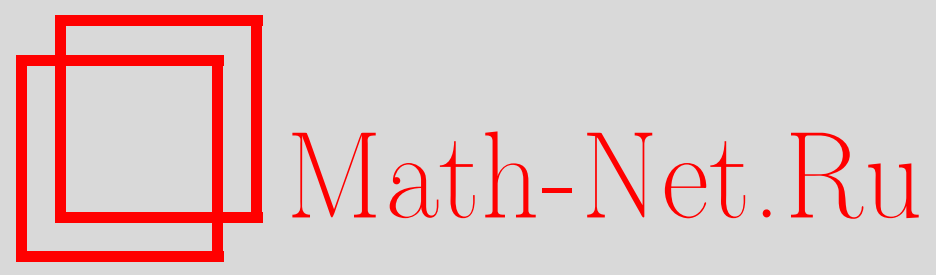

Обцероссийский математический портал 
К. Н. Кобаненко, О продолжении обобщенно-липшицевых отображений, Матем. заметки, 1998, том 63, выпуск $5,789-792$

DOI: https://doi.org/10.4213/mzm1346 
Использование Общероссийского математического портала Math-Net.Ru подразумевает, что вы прочитали и согласны с пользовательским соглашением http://www. mathnet.ru/rus/agreement

Параметры загрузки:

IP : 54.209 .52 .79

26 апреля 2023 г., 14:49:36 


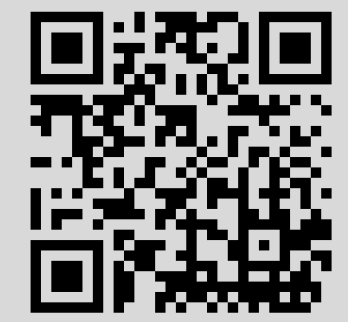




\section{О ПРОДОЛЖЕНИИ ОБОБЩЕННО-ЛИПШИЦЕВЫХ ОТОБРАЖЕНИЙ}

\section{К. Н. Кобаненко}

Пусть $X$ - локально выпуклое топологическое пространство с гауссовой мерой $\mu, E$ - воспроизводящее ядро меры $\mu, Y$ - банахово пространство с нормой $|\cdot|$, a $f: X \rightarrow Y$ - измеримое отображение.

Согласно полученному в [1] результату (являющемуся, в частности, обобщением известной теоремы Ферника [2]) включение $\exp (c|f|) \in L^{1}(\mu)$ для достаточно малых $c>0$ вытекает из следующего свойства $f$ :

$$
|f(x+h)-f(x)| \leqslant \lambda\|h\|_{E} \quad \forall h \in E .
$$

(C) К. Н. Кованенко 
В связи с этим появилось несколько интересных задач, одной из которых является задача о продолжении измеримого отображения, заданного на измеримом подмножестве в $X$ и обладающего на нем свойством (1), на все пространство $X$ с сохранением измеримости и этого свойства с той же константой. Следует отметить, что болшое количество работ было посвящено изучению более специальной задачи продолжения липшицева (или гёльдерова) отображения $f$, заданного на подмножестве $A$ нормированного (или даже метрического) пространства $X$ и принимающего значения в нормированном пространстве $Y$. Один из первых результатов в этом направлении получен Макшейном в [3], где доказано, что всякая числовая липшицева функция $f$ на произвольном подмножестве метрического пространства $X$ продолжается с сохранением константы Липшица на все пространство (соответствующее продолжение задается простой явной формулой). Для многомерных отображений положение более сложное. Например, возможны ситуации (см. [4]-[6]), когда $X$ и $Y$ - банаховы пространства, но некоторое отображение $f: A \rightarrow Y$ вообще не имеет липшицевых продолжений (даже и без ограничений на константу Липшица). Для некоторых пар пространств $X$ и $Y$ продолжение существует всегда, причем без увеличения константы Липшица, а для некоторых пар пространств продолжение хотя и всегда возможно, но не всегда существует с прежней константой Липшица (см. [4], [5]). Один из наиболее известных положительных результатов - теорема из [7], согласно которой всякое липшицево отображение $f$, заданное на подмножестве гильбертова пространства $X$ и принимающее значения также в гильбертовом пространстве $Y$, имеет липшицево с той же константой продолжение на все $X$. Однако, в отличие от скалярных функций такое продолжение не описьвается явной формулой, а лишш выводится из одного свойства пересечений выпуклых тел. Приводимьй ниже результат представляет собой обобщение упомянутой теоремы Макшейна для описанной выше ситуации. По-видимому, справедлив и соответствующий аналог теоремы [7] для бесконечномерных отображений, хотя автору не удалось это показать даже для отображений в $\mathbb{R}^{2}$ (для рассмотрения бесконечномерного случая достаточно было бы доказать возможность осуществлять $E$-липшицевы продолжения без увеличения константы Липшица для отображений в $\mathbb{R}^{n}$ ).

Напомним, что образы полных сепарабельных метрических пространств при непрерывных отображениях в хаусдорфовы пространства называются суслинскими пространствами (см. [8]). Известно, что таковы и непрерывные образы борелевских множеств в полных сепарабельных метрических пространствах. Болшшинство локально выпуклых пространств, встречающихся в приложениях, являются суслинскими (хотя при этом они могут и не быть метризуемыми, как, например, пространства $\mathscr{S}^{\prime}\left(\mathbb{R}^{n}\right), \mathscr{D}\left(\mathbb{R}^{n}\right), \mathscr{D}^{\prime}\left(\mathbb{R}^{n}\right)$ и сепарабельные банаховы пространства со слабой топологией).

Через $\mathscr{E}(T)$ обозначим $\sigma$-алгебру, порожденную суслинскими подмножествами топологического пространства $T$.

Теорема. Пусть $X$ - суслинское топологическое векторное пространство (например, сепарабельное пространство фреше), $E \subset X$ - линейное подпространство, наделенное нормой $\|\cdot\|_{E}$ такой, что шар $U=\left\{h \in E:\|h\|_{E} \leqslant 1\right\}$ - суслинское мнохсество в $X, A \subset X$ - некоторое суслинское множсество, а $f: A \rightarrow \mathbb{R}^{1}$ - функция, которая обладает следующими свойствами:

а) множества $\{x \in A: f(x)>c\}-$ суслинские,

б) для всех $h \in E$ u $x \in A$ таких, что $x+h \in A$, справедливо неравенство

$$
|f(x+h)-f(x)| \leqslant \lambda\|h\|_{E} .
$$

Тогда функиию $f$ можно продолжить до функиии $F: X \rightarrow \mathbb{R}^{1}$, обладающей свойством а) (в частности, универсально измеримой) и удовлетворяющей неравенству (1).

ДоКАЗАТЕЛЬСтво. Очевидно, что функция, задаваемая формулой

$$
F(x)= \begin{cases}\sup _{y \in A_{x}} f(y)-\lambda\|x-y\|_{E}, & \text { если } A_{x} \neq \varnothing, \\ 0, & \text { если } A_{x}=\varnothing,\end{cases}
$$

где $A_{x}=(x+E) \cap A$, обладает свойством (1) и при $x \in A$ совпадает с $f(x)$. Необходимо доказать только, что множества $\{x: F(x)>c\}$ - суслинские (откуда вытекает, что функция $F$ измерима 
относительно $\mathscr{E}(X))$. Отметим, что приведенная формула является непосредственным обобщением формулы Макшейна [3]. Ясно, что в определении $F$ при взятии супремума можно заменить $A_{x}$ на $A$. Таким образом, $F(x)=0$, если $x$ не входит в множество $X_{0}=A+E$, являющееся суслинским, как образ суслинского множества $A \times E$ в $X \times X$ при непрерьвном отображении $(x, y) \mapsto x+y$ (отметим, что из того, что единичный шар из $E$ - суслинское множество в $X$, вытекает, что таковы же и все шары из $E$, а также и само $E$ ).

Пусть $Z=\{-\infty\} \cup \mathbb{R}^{1} \cup\{+\infty\}$ - двухточечная компактификация $\mathbb{R}^{1}$. Продолжим $\|\cdot\|_{E}$ на $X$, положив $\|x\|_{E}=+\infty$ при $x \notin E$. Определим отображение $\|x-y\|: X \times X \rightarrow Z$ следующей формулой:

$$
\|x-y\|= \begin{cases}\|x-y\|_{E}, & \text { если } x-y \in E, \\ +\infty, & \text { если } x-y \notin E .\end{cases}
$$

Для всякого числа $r$ множество $\left\{x \in X:\|x\|_{E} \leqslant r\right\}$ - суслинское, поскольку оно совпадает с шаром радиуса $r$ в $E$. В силу непрерьвности отображения $X \times X \rightarrow X,(x, y) \mapsto x-y$, множество $\left\{(x, y) \in X \times Y:\|x-y\|_{E} \leqslant r\right\}$ суслинское для всякого числа $r$. Значит, для всех $c \in \mathbb{R}^{1}$ суслинским является и множество $\left\{(x, y) \in X_{0} \times A: f(y)-c\|x-y\|_{E}>0\right\}$, являющееся объединением по всем рационалным $r$ суслинских множеств

$$
\left\{(x, y) \in X_{0} \times A: f(y)>r\right\} \cap\left\{(x, y) \in X_{0} \times A: r>c\|x-y\|_{E}\right\} .
$$

Положим $G(x, y)=f(y)-\lambda\|x-y\|_{E}$. Очевидно, что

$$
\left\{x \in X_{0}: F(x)>c\right\}=p\left(\left\{(x, y) \in X_{0} \times A: G(x, y)>c\right\}\right),
$$

где $p: X \times X \rightarrow X$-естественная проекция на первый сомножитель. Таким образом, множество $\left\{x \in X_{0}: F(x)>c\right\}$ суслинское. Теорема доказана.

Ситуация в случае, когда $Y$ - гильбертово пространство, оказьвается более сложной, так как не удается конструктивно построить искомое отображение, как это получилось в доказанной теореме. Например, естественная идея брать в качестве $F(x)$ точку из $\bigcap_{x \in A} B\left(f(y), c\|x-y\|_{E}\right)$, ближайшую к 0 , как легко показать, не приводит к цели.

ЗАмечАниЕ 1. Как явствует из доказательства теоремы, аналогичное утверждение справедливо и в случае, когда $X$ - суслинское пространство (не обязательно линейное), $A \subset X$ - суслинское подмножество $X$, причем на $X$ задана такая квази-метрика $\rho: X \times X \rightarrow \mathbb{R}^{1} \cup\{+\infty\}$, что множества $\{(x, y) \in X \times Y: \rho(x, y) \leqslant r\}$ суслинские, а функция $f: A \rightarrow \mathbb{R}^{1} \rho$-липшицева, т.е. $|f(x)-f(y)| \leqslant \lambda \rho(x, y)$ для всех $x, y \in A$ (при этом неравенство считается верным, если $\rho(x, y)=+\infty)$.

Аналогичное утверждение справедливо и в случае, если вместо классических суслинских множеств рассматривать абстрактные классы, полученные с помощью $A$-операции из каких-либо заранее заданных классов множеств.

ЗАмечание 2. Как показано в [9], для всякой меры Радона $\mu$ на $X$, квазиинвариантной по направлениям из $E$, и всякого борелевского отображения $F$ из $X$ в $E$, которое липшицево вдоль $E$, $\mu$-почти всюду существует производная Гато вдоль $E$. Это, с одной стороны, означает, что исходное отображение в теореме выше в определенном смысле обладает свойством дифференцируемости, а, с другой стороны, естественно приводит к вопросу о возможном распространении этой теоремы на бесконечномерные отображения.

ЗАмЕчАниЕ 3 . В недавней статье [10] получено близкое (но более слабое утверждение): если $f$ - функция, измеримая относительно гауссовской меры $\mu$ на сепарабельном банаховом пространстве $X$, заданная на $\mu$-измеримом множестве $A$ и удовлетворяющая на нем условию Липшица вдоль пространства Камерона-Мартина $E$, то существует $\mu$-измеримая функция на всем $X$, удовлетворяющая тому же условию и совпадающая с $f \mu$-почти всюду. Это утверждение вытекает из доказанной выше теоремы, поскольку $f$ имеет борелевскую модификацию, удовлетворяющую тому же условию на борелевском множестве $B \subset A$ с $\mu(B)=\mu(A)$. Из доказанной теоремы вытекает, что то же самое верно и в случае, когда $X$ - локально выпуклое пространство с радоновской гауссовской мерой $\mu$ (в этом случае согласно [11] мера $\mu$ сосредоточена на суслинском линейном подпространстве). 


\section{СПИСОК ЦИТИРОВАННОЙ ЛИТЕРАТУРЫ}

1. Cirel'son B.S., Ibragimov I. A., Sudakov V. N. // Lecture Notes in Math. 1976. V. 550. P. 20-41. 2. Fernique X. // C. R. Acad. Sci. Paris. Sér. I. Math. 1970. V. 270. P. 1698-1699. 3. McShane E. J. // Bull. Amer. Math. Soc. 1934. V. 40. P. 837-842. 4. Wells J. H., Williams L. R. Embeddings and Extensions in Analysis. Berlin: Springer, 1975. 5. Данцер Л ., Грюнбаум Б., Кли В. Теорема Хелли. М.: Мир, 1968. 6. Царьков И. Г. // Матем. заметки. 1993. T. 54. № 3. C. 123-140. 7. Valentine F. // Bull. Amer. Math. Soc. 1943. V. 49. P. 100-108. 8. Фeдерер Г. Геометрическая теория меры. М.: Наука, 1987. 9. Bogachev V., Mayer-Wolf E. // Potential Anal. 1973. V. 5. №1. P. 23-30. 10. Ustunel A. S., Zakai M. Extension of Lipschitz Functions on Wiener Space. Preprint, 1995. 11. Цирельсон Б. С. // Записки научн. семин. ЛОМИ. 1977. V. 55. Р. 35-63; Дополнение // Записки научн. семин. ЛОМИ. 1977. V. 72. P. 202-211.

Московский государственный университет им. М. В. Ломоносова

Исправленньй вариант 